\title{
Role of plasmapheresis in primary biliary cirrhosis
}

\author{
L B COHEN, E P AMBINDER, A M WOLKE, S P FIELD, AND F SCHAFFnER
}

From the Departments of Medicine and Neoplastic Diseases, Mount Sinai School of Medicine, The City

University of New York, New York, USA

SUMMARY Five patients with primary biliary cirrhosis and prolonged cholestasis underwent intensive plasmapheresis. The indications for plasmapheresis included intractable pruritus or hypercholesterolemia and xanthomatous neuropathy. Patients noted a rapid improvement of pruritus and fatigue which was sustained as long as plasmapheresis was continued. Cholesterol levels were lowered an average of $10.3 \mathrm{mmol} / \mathrm{l}$ and xanthomata were reduced in three of four patients. Two patients with painful neuropathy caused by xanthomata experienced relief of this symptom. The liver and spleen size were not affected by plasmapheresis, and activities of aminotransferases, alkaline phosphatase and titres of mitochondrial antibody remained unchanged. We conclude that plasmapheresis has a role in the therapeutic management of patients with advanced primary biliary cirrhosis who are disabled by the complications of pruritus, xanthomatous neuropathy, or hypercholesterolemia with xanthoma formation.

The treatment of primary biliary cirrhosis is directed at relief of symptoms. Complications of the disease are the result of prolonged cholestasis: pruritus, jaundice, hypercholesterolemia with xanthoma, and xanthomatous neuropathy, fat malabsorption, and osteopenia. Therapy is not effective for the hypercholesterolemia, and the pruritus, which responds early to the bile salt-sequestering resin, cholestyramine, may with time become refractory to such therapy. Based on prior reports of the efficacy of plasmapheresis in hypercholesterolemia, ${ }^{12}$ xanthomatous neuropathy, ${ }^{2}$ and pruritus, ${ }^{34}$ we undertook a study of plasma exchange in five patients with primary biliary cirrhosis to answer the following questions: (1) can plasmapheresis effect sustained lowering of serum cholesterol levels with improvement of xanthomas and painful xanthomatous neuropathy? (2) can plasmapheresis relieve pruritus unresponsive to cholestyramine? and (3) can plasmapheresis improve the laboratory parameters of liver disease?

\section{Methods}

PATIENTS

Five patients with primary biliary cirrhosis were studied, four women and one man, with a mean age

Address for correspondence: Lawrence B Cohen, MD, 920 Park Avenue, New York. New York 10028, USA.

Received for publication 18 May 1984 of 44 years. The mean duration of disease was 10 years. The diagnosis of primary biliary cirrhosis was based on the following criteria: (1) alkaline phosphatase more than twice upper limit of normal, (2) the presence of mitochondrial antibody in the serum, (3) a compatible histologic appearance of a liver biopsy specimen, (4) normal extrahepatic bile ducts by endoscopic retrograde or operative cholangiography. The indications for initiation of plasmapheresis included intractable pruritus (five), and hypercholesterolemia (five) with xanthoma formation (four) and/or xanthomatous neuropathy (two). Additional findings which were noted before plasmapheresis included jaundice (five), hepatomegaly (three), fatigability (four), nausea (five), Sjögren's syndrome (four), and Raynaud's phenomenon (one). All patients had advanced disease at stage III or IV. ${ }^{5}$ The administration and dosage of other medications were held constant during the study period. All patients were receiving colchicine (four) and/or penicillamine (three).

\section{TECHNIQUE}

Plasmapheresis was performed using the Haemonetics Model 30 blood cell separator, using standard plasmapheresis technique. At each visit, $500-2000 \mathrm{ml}$ of plasma was removed during a two to three hour session and replaced with $1000 \mathrm{ml}$ of normal saline and 5\% albumin. Patients underwent apheresis three times a week for the first two to 
three weeks until a clinical response was noted. Maintenance plasmapheresis was continued at one to two week intervals. A questionnaire was administered to patients before initiation of plasmapheresis, and every three months to assess the presence of pruritus, fatigability, dryness of the eyes or mouth, Raynaud's phenomenon, nausea and vomiting. Clinical scoring was as follows: $0=$ absent,$+1=$ mild,$+2=$ moderate,$+3=$ severe, and $+4=$ disabling. The following physical findings were evaluated: hepatomegaly, splenomegaly, xanthomata, and peripheral neuropathy. Laboratory parameters including automated chemistry screen, immunoglobulins and mitochondrial antibody were studied serially. Immune complexes were estimated using a solid phase C1q-binding assay.

\section{Results}

Patients underwent plasmapheresis a mean of 63 times during a mean study period of 26 months (range, 20-37 months). An average of 95 litres was exchanged per patient during the study period. The clinical results of plasmapheresis are presented in Table 1. All patients experienced marked relief of pruritus, less fatigability accompanied by an improved sense of well-being, and a reduction in nausea. Xanthomatous neuropathy was relieved in both patients with this symptom. In addition, all four patients with Sjögren's syndrome had an improvement and Raynaud's phenomenon was improved in the one patient so affected. Xanthomata were reduced in three of four patients, but liver and spleen size were not affected.

The serum cholesterol level was most significantly affected by plasmapheresis, reduced by a mean of $10 \cdot 3 \mathrm{mmol} / \mathrm{l}$ (range, 5.7-16.7 $\mathrm{mmol} / \mathrm{l}$ ) (Table 2).
This reduction was sustained with continued plasma exchange. The alkaline phosphatase and aminotransferase activities, bilirubin, and mitochondrial antibody titres were not affected by plasmapheresis. Bilirubin concentrations dropped 20-40\% immediately after each treatment but were back to pretreatment levels within a week. Over the entire period of study the gradual rise of bilirubin expected did not occur. Immune complexes were detected in two patients studied for their presence, and were cleared by plasmapheresis.

\section{Discussion}

The aetiology of primary biliary cirrhosis is unknown but is thought to involve an immunologic derangement. The presence of circulating autoantibodies in greater than $90 \%$ of patients and the frequent coexistence of Sjögren's syndrome, Raynaud's phenomenon, and thyroiditis, ${ }^{6}$ have raised the question of autoimmune origin. No specific therapy for the disease exists.

Treatment currently is directed at alleviating symptoms. The most troublesome symptom is pruritus, which usually responds to cholestyramine. ${ }^{7}$ Some patients with advanced disease, however, become refractory to this drug as cholestasis becomes more severe. Also troublesome is the severe hypercholesterolemia which occurs, with its sequalae of xanthomata, painful xanthomatous neuropathy, ${ }^{8}$ and possibly accelerated atherosclerosis. ${ }^{9}$ Fatigability may also be severe and no therapy improves this.

Our study confirms a previous report ${ }^{10}$ that plasmapheresis has a role in the palliative management of primary biliary cirrhosis when complicated by these symptoms. Patients experience a dramatically improved sense of well-being, relief of

Table 1 Signs and symptoms

\begin{tabular}{|c|c|c|c|c|c|c|c|c|c|c|}
\hline & \multicolumn{10}{|c|}{ Patients } \\
\hline & \multicolumn{2}{|l|}{$R F$} & \multicolumn{2}{|l|}{$S D$} & \multicolumn{2}{|l|}{$M D$} & \multicolumn{2}{|l|}{$A R$} & \multicolumn{2}{|l|}{$C G$} \\
\hline & Pre & Post & Pre & Post & Pre & Post & Pre & Post & Pre & Post \\
\hline Fatigue & $3+$ & $1+$ & $2+$ & $1+$ & 0 & 0 & $2+$ & $1+$ & $2+$ & $1+$ \\
\hline Pruritus & $3+$ & 0 & $3+$ & $1+$ & $3+$ & 0 & $3+$ & $1+$ & $3+$ & $1+$ \\
\hline Nausea & $1+$ & 0 & $1+$ & 0 & $3+$ & 0 & $1+$ & 0 & $1+$ & 0 \\
\hline Sjögren's syndrome & $2+$ & 0 & $1+$ & 0 & 0 & 0 & $2+$ & 0 & $1+$ & 0 \\
\hline Raynauds phenomenon & 0 & 0 & 0 & 0 & 0 & 0 & 0 & 0 & $3+$ & 0 \\
\hline Liver size $(\mathrm{cm})$ & 18 & 13 & 10 & 10 & 11 & 11 & 16 & 14 & 16 & 16 \\
\hline Spleen size ( $\mathrm{cm}$ below costal margin) & 4 & 6 & 0 & 1 & 1 & 1 & 5 & 7 & 0 & 0 \\
\hline Xanthomata & $3+$ & 0 & $2+$ & $2+$ & 0 & 0 & $3+$ & $1+$ & $2+$ & 0 \\
\hline Painful neuropathy & 0 & 0 & 0 & 0 & 0 & 0 & $3+$ & 0 & $4+$ & 0 \\
\hline Duration of therapy (months) & & 22 & & 28 & & 18 & & 26 & & 37 \\
\hline
\end{tabular}




\begin{tabular}{|c|c|c|c|c|c|c|c|c|c|c|}
\hline & \multicolumn{10}{|c|}{ Patients } \\
\hline & \multicolumn{2}{|l|}{$R F$} & \multicolumn{2}{|l|}{$S D$} & \multicolumn{2}{|l|}{$M D$} & \multicolumn{2}{|l|}{$A R$} & \multicolumn{2}{|l|}{$C G$} \\
\hline & Pre & Post & Pre & Post & Pre & Post & Pre & Post & Pre & Post \\
\hline AST (nl, 1-50 U/l) & 186 & 137 & 102 & 131 & 108 & 72 & 68 & 74 & 114 & 137 \\
\hline AST (nl, 1-53 U/l) & 180 & 114 & 157 & 156 & 185 & 90 & 88 & 67 & 88 & 113 \\
\hline Alkaline phosphatase (nl, 30-90 U/l) & 1273 & 1004 & 425 & 682 & 978 & 1255 & 1025 & 846 & 942 . & 835 \\
\hline Total bilirubin $(\mu \mathrm{mol} / \mathrm{l})$ & 274 & 222 & 274 & 222 & 325 & 239 & 205 & 239 & 85 & 68 \\
\hline $\operatorname{IgM}(\mathrm{g} / \mathrm{l})$ & 8 & 3.4 & $7 \cdot 2$ & $10 \cdot 5$ & $2 \cdot 7$ & $4 \cdot 3$ & $1 \cdot 6$ & $7 \cdot 6$ & 10 & $11 \cdot 2$ \\
\hline Mitochondrial antibody (titre) & $1: 320$ & $1: 320$ & $1: 160$ & $1: 160$ & $1: 160$ & $1: 320$ & $1: 80$ & $1: 320$ & $1: 80$ & $1: 640$ \\
\hline Cholesterol $(\mathrm{mmol} / \mathrm{l})$ & $14 \cdot 7$. & 9 & 22.9 & $7 \cdot 4$ & $11 \cdot 3$ & $4 \cdot 5$ & $15 \cdot 7$ & $9 \cdot 1$ & $25 \cdot 3$ & $8 \cdot 6$ \\
\hline
\end{tabular}

pruritus, resorption of xanthomata, and elimination of the pain caused by xanthomatous neuropathy, accompanied by a reduction in serum cholesterol. Additionally, the symptoms of Sjögren's syndrome and Raynaud's phenomenon appear to be reduced.

The mechanism of these effects is removal from the body of cholesterol and bile salts (or other pruritogens) which are normally excreted via the bile. Relief of the Sjögren's or Raynaud's symptoms may depend upon removal of other substances - for example, immune complexes, but this is not clear. The effect of plasmapheresis on liver function and on the long term prognosis of primary biliary cirrhosis cannot be assessed from this study. If immune aberrations are aetiologic as suggested by Sherlock ${ }^{11}$ and others, ${ }^{12}$ long term apheresis, by removing autoantibodies or immune complexes, could possibly alter the basic disease mechanism; this remains to be shown. What does seem clear is that chronic plasmapheresis has a role in improving the quality of life for patients with primary biliary cirrhosis with intractable pruritus and/or the complications of protracted hypercholesterolemia. The rate of progression of the disease seems to be slowed as life expectancy is otherwise very short in patients with primary biliary cirrhosis and extreme rises in bilirubin. ${ }^{13}$

Over a three year period encompassing 317 procedures, we have found plasmapheresis to be a safe therapeutic modality. Mild urticaria and transient hypotension have occurred several times but always with rapid response to fluids, albumin, and adrenaline. In contrast, Rubenstein ${ }^{14}$ recently reported three cases of anaphylaxis in 22 patients receiving therapeutic plasma exchange, manifested by urticaria, bronchospasm, and hypotension. Fatal pulmonary microvascular occlusion developed in an additional patient after an apheresis procedure. Thus, caution must be exercised, and patients closely followed after initiating plasmapheresis.

\section{References}

1 Thompson GR. Plasma exchange for hypercholesterolaemia. Lancet 1981; 1: 1246-8.

2 Turnberg LA, Mahoney MP, Gleeson MH, Freeman CB, Gowenlock AH. Plasmapheresis and plasma exchange in the treatment of hyperlipaemia and xanthomatous neuropathy in patients with primary biliary cirrhosis. Gut 1972; 13: 976-81.

3 Lauterburg BH, Pineda AA, Burgstaler EA, Taswell HF, Dickson ER, Carlson GL. Treatment of pruritus of cholestasis by plasma perfusion through USP. charcoal-coated glass beads. Lancet 1980; 2: 53-5.

4 Keeling PWN, Bull J, Kingston P, Thompson RPH. Plasma exchange in primary biliary cirrhosis. Postgrad Med J 1981; 57: 433-5.

5 Schaffner F, Popper H. Clinical-pathologic relations in primary biliary cirrhosis. In: Popper H, Schaffner F, eds. Progress in liver diseases. Vol 7. New York: Grune and Stratton, 1982: 529-54

6 Clarke AK, Galbraith RM, Hamilton EBD, Williams $\mathrm{R}$. Rheumatic disorders in primary biliary cirrhosis. Ann Rheum Dis 1978; 37: 42-7.

7 Schaffner F, Klion FM, Latuff AJ. The long term use of cholestyramine in the treatment of primary biliary cirrhosis. Gastroenterology 1965; 48: 293-8.

8 Thomas PK, Walker JG. Xanthomatous neuropathy in primary biliary cirrhosis. Brain 1965; 88: 1079-88.

9 Wolke A, Brooks K, Sacks H, Schaffner F. Extrahepatic malignancy and atherosclerosis in primary biliary cirrhosis. [Abstract] Gastroenterology 1982; 82: 1212.

10 Eriksson S, Lindgren S. Plasma exchange in primary biliary cirrhosis. [Letter] $N$ Engl J Med 1981; 302: 809.

11 Thomas HC, Potter BJ, Sherlock S. Is primary biliary cirrhosis an immune complex disease? Lancet 1977; 2: 1261-3.

12 Wands KR, Dienstag JL, Bhan AK, Feller ER, Isselbacher KJ. Circulating immune complexes and complement activation in primary biliary cirrhosis. $N$ Engl J Med 1978; 298: 233-7.

13 Shapiro JM, Smith H, Schaffner F. Serum bilirubin: a prognostic factor in primary biliary cirrhosis. Gut 1979; 20: $137-40$. 


\section{Forthcoming Scientific Meetings of the British Society of Gastroenterology}

Brighton 20-22 March 1985

Newcastle-upon-Tyne 18-20 September 1985

Lancaster 9-11 April 1986

Cardiff 24-26 September 1986

London 15-18 September 1987

For details of registration please contact: The Administrative Secretary, British Society of Gastroenterology, Rayne Institute, 5 University Street, London, WC1E 66JJ. Tel: (44: country code) 01-387-3534. 